\title{
Ruptured Gastroduodenal Artery Aneurysm Presenting As Upper Gastrointestinal Bleed
}

\author{
SM Ikhwan ${ }^{1}$, WM Mokhzani $^{1}$, AB Norzila ${ }^{2}$, A Shafiee $^{2}$, S Hassan $^{3}$, Z Zaidi $^{1}$ \\ ${ }^{1}$ Dept. of Surgery, Hospital Universiti Sains Malaysia, Kubang Kerian, Kelantan. \\ ${ }^{2}$ Dept. of Radiology, Hospital Universiti Sains Malaysia, Kubang Kerian, Kelantan. \\ ${ }^{3}$ Endoscopy Unit, Hospital Universiti Sains Malaysia, Kubang Kerian, Kelantan.
}

\begin{abstract}
Gastroduodenal artery (GDA) aneurysm is a very rare condition out of all vascular aneurysms. It represents only $1.5 \%$ of reported splanchnic artery aneurysms. This is a case of a 37-year-old man who presented with upper gastrointestinal bleeding.oesophagogastroduodenoscopy revealed an ulcer at first part of duodenum.During intervention towards the ulcer, it was massively bleed and unable to be controlled by endoscopic mean. Emergency angiogram was done and suprisingly showed an aneurysm of the gastroduodenal artery which ran below the ulcer.It was successfully embolized and thebleeeding arrested.The discussion is directed towards detection of rare causes of upper gastrointestinal bleeding and the best modalities to treat this condition either by endoscopic mean, interventional radiology or surgery.
\end{abstract}

Keyword : gastroduodenal artery aneurysm ; upper gastrointestinal bleeding

\section{Introduction}

Aneurysms of the splanchnic arteries are rare with splenic artery aneurysm being the most common location (10.4\%), while gastroduodenal artery (GDA) aneurysm represents only $1.5 \%$ of of more than 3000 reported splanchnic artery aneurysms reported since 1960's. ${ }^{1,2}$ Most of the GDA aneurysms described in the literature are pseudoaneurysms associated with pancreatitis. ${ }^{3}$ Rupture of GDA aneurysm is usually a dramatic manifestation of hemorrhagic shock associated with high mortality. ${ }^{4}$ We present a case of a GDA aneurysm rupture that was diagnosed incidentally during endovascular intervention for a patient with upper GI bleed.

\section{Case Report}

A 37 year old male presented to the emergency department with sudden episode of massive fresh hematemesis. He had no previous history of upper GI bleed. He is an active intravenous drug user and is known positive for retroviral disease but not on anti-viral therapy. Initial assessment revealed grade II hypovolemic shock as evidenced by clinical pallor, hypotension and tachycardia. Initial resuscitation was carried out with high-flow mask oxygen, large calibre intravenous access and rapid fluid replacement. Urgent group and cross match was done and packed cell transfusion was started in the emergency room. Urgent upper endoscopy was arranged and the patient was promptly transferred to the endoscopy suite with stable vital signs.

Upper endoscopy showed normal esophagus and normal stomach mucosa. Upon intubation of the duodenum, a Forrest IIa ulcer was visible in the first part of duodenum. There was no evidence of active bleeding. The plan was for injection with noradrenaline and clipping of the ulcer. However, immediately upon injecting the ulcer, the endoscopic view was immediately obscured by massive amount of blood gushing out from the duodenal wall. The scope was reversed, Ryles tube inserted and urgent CT angiography was arranged with planned embolization of the bleeding vessel.

Preliminary CT angiogram showed presence of single aneurysm in the celiac artery with a complex aneurysm of the gastroduodenal artery (Figure 1). The location of the gastroduodenal artery aneurysm was consistent with the endoscopic finding of the duodenal ulcer.

Embolization of the gastroduodenal aneurysm was done using coil. However, there were difficulties in achieving this goal as the angulation of the vessels was deemed 'challenging' by the interventional radiologist (Figure 2). The procedure was successful after 3 hours and the patient was nursed in ICU. However due to massive blood transfusions, patient developed complication of severe acute respiratory distress syndrome .The patient succumbed to death 24 hours later due to multiorgan failure. 


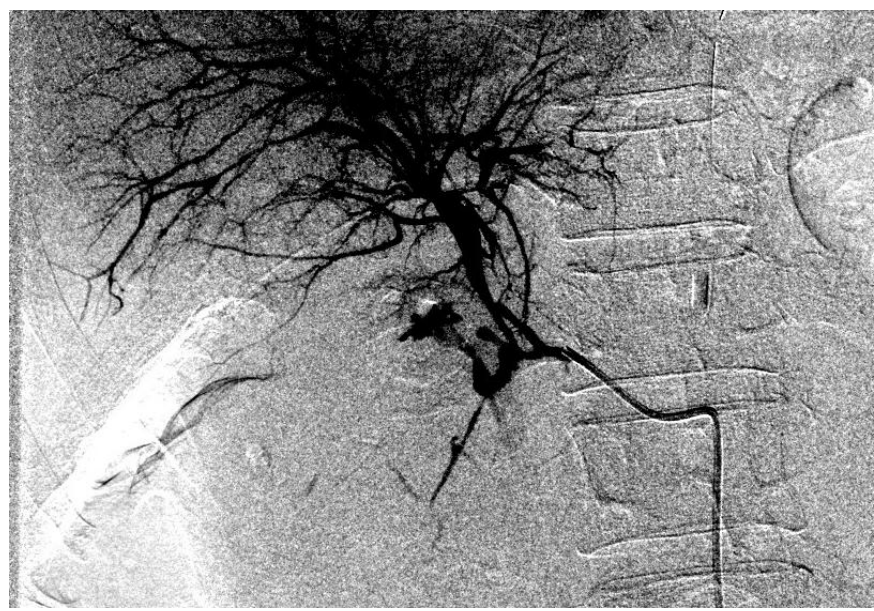

Figure1 .Selective Pre-Embolization run of Common Hepatic Artery shows aneurysmal dilatation at proximal portion of Gastroduodenal artery with contrast extravasation into the small bowel
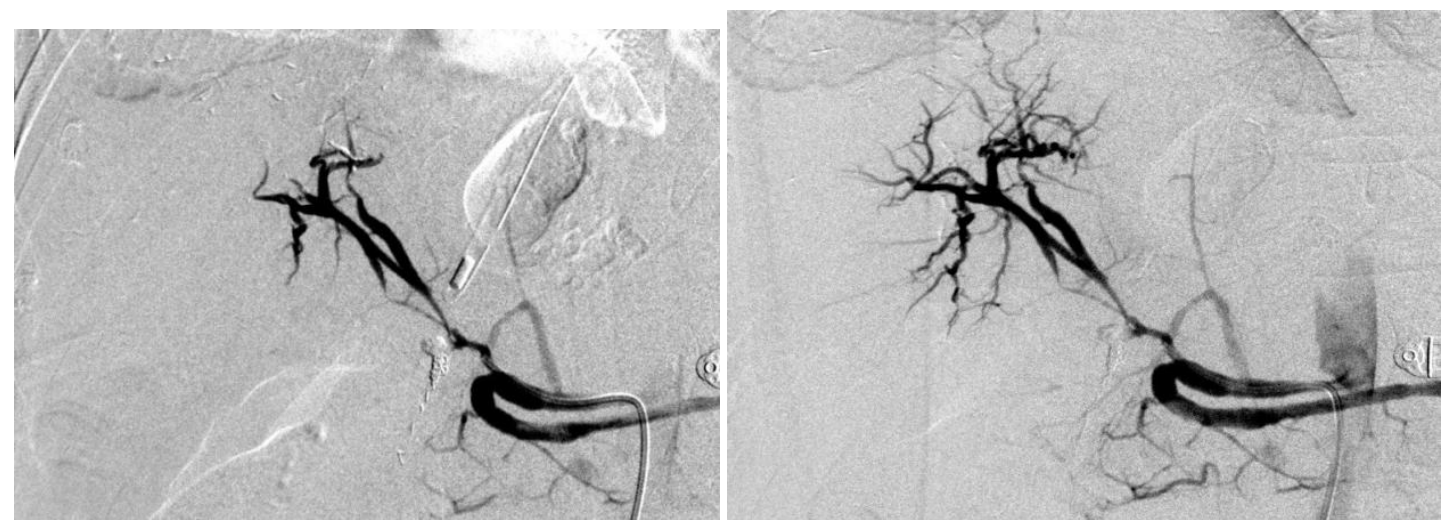

Figure 2. Selective Post Embolization run of Common Hepatic artery shows successful embolization of the gastroduodenal artery.

\section{Discussion}

Splanchnic artery aneurysms are rare, with an incidence of $0.01-0.2 \%$ in routine autopsies. ${ }^{5}$ True gastroduodenal artery aneurysms are uncommon as it accounts for only $1.5 \%$ of around 3000 splanchnic artery aneurysms documented in the literature. ${ }^{2}$

It most often occurs as a complication in both acute and chronic pancreatitis. ${ }^{6}$ Periarterial inflammation by pancreatic enzymes secondary to pancreatitis accounts for GDA pseudoaneurysms while possible etiologies for true GDA aneurysms include atherosclerosis, mycotic aneurysms, autoimmune diseases such as Wegener's granulomatosis and systemic lupus erythematosus, and an extremely rare condition of congenital absence of

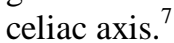

The underlying etiology of the GDA aneurysm in this presentation remains speculative but possibilities include chronic pancreatitis as retrospectively history from relative also reveal significant alcohol consumption. Underlying mycotic aneurysm of the visceral artery is also a possibility in view of the significant history of intravenous drug abuse and his retroviral status.

The clinical presentation of GDA aneuryms is variable and may include palpable pulsatile mass, pressure effect on the surrounding structures causing pain, jaundice, hemorrhage or gastric outlet obstruction. As application of more advanced imaging modalities in evaluating intra abdominal mass increases, the incidental diagnosis of these otherwise assymptomatic aneurysms have also increased. ${ }^{8}$

Many of the assymptomatic splanchnic artery aneurysms are detected incidentally on imaging. Plain abdominal radiograph may show curved calcifications in the presence of underlying calcified vessels. Vascular mass may be evident on ultrasonography, computerized tomography scan (CT) or magnetic resonance imaging (MRI). Presentation as upper GI bleed due to aneurysm rupture is rare. ${ }^{9}$

The diagnosis is usually established by means of selective arteriogram and CT angiography. ${ }^{9}$ Early detection and effective treatment are necessary to improve the prognosis of these aneurysms. ${ }^{10}$ Management options include observation for assymptomatic small aneurysms, surgical repair and endovascular treatment with catheter based embolization. In selected patients, stent-graft deployment have been described. ${ }^{11}$ 
Surgery is the conventional treatment of splanchnic artery aneurysm, with a 5\% mortality rate during elective repair and a significantly higher $40 \%$ mortality of ruptured aneurysm. ${ }^{1}$ Open surgical repair of these aneurysms is increasingly being replaced by endovascular embolization due to its lower morbidity and mortality and high success rate. ${ }^{11}$ The accepted endovascular approach includes coil embolization of the aneurysmal lumen, the proximal and distal aneurysmal neck, or both. There are also anecdotal reports of endovascular stent-graft repair in splanchnic artery aneurysms..$^{11}$

Possible advantages of endovascular techniques include accurate anatomical localization, ability to be performed under local anaesthesia, and thus avoiding the risks associated with open surgical repair such as ileus, intra-abdominal sepsis, bleeding, wound infections and prolonged hospital stay. ${ }^{8}$

Limitations of endovascular approach includes ruptured aneurysms not contained with embolization and giant sized aneurysms with involvement of surrounding structures. ${ }^{12}$

Reported complications dueto endovascular embolization of splanchnic artery aneurysms include end organ infarction, aneurysm recurrence, contrast nephropathy, embolism and access site hematoma. ${ }^{8}$ It is therefore crucial for patients who underwent successful embolization of the aneurysms to be assessed with repeat imaging during follow up visits.

\section{Conclusion}

This case illustrates the point that management of upper GI bleed in high-risk patients should take into consideration the possibility of uncommon etiologies such as ruptured splanchnic artery aneurysms. Although a rare entity, ruptured GDA aneurysm is associated with significant mortality and therefore requires a high index of suspicion in diagnosis. Published literature on this topic favors endovascular embolization of the aneurysm but open surgical repair remains a viable option in cases where non-operative approach fails or the size of the aneurysm is not favorable for embolization.

\section{References}

[1]. Stanley JC, Zelenock GB. Splanchnic artery aneurysms. In: Rutherford RB, editor. Vascular Surgery. 4th ed. Philadelphia: WB Saunders; 1995. p. 1124-81.

[2]. R.R. Babb. Aneurysm of the splenic artery. Arch Surg, 111 (1976), pp. 924-925.

[3]. R. Schmitt, A. Cavallaro, W. Bautz. Segmental embolization of the gastroduodenal artery in a case of a perforated pseudoaneurysm and gastrointestinal massive bleeding. Rontgenpraxis, 55 (4) (2004), pp. 147-151.

[4]. T. Suzuki, H. Ishida, T. Komatsuda, J. Oyake, T. Miyauchi, J. Heianna et al. Pseudoaneurysm of the gastroduodenal artery ruptured into the superior mesenteric vein in a patient with chronic pancreatitis. J Clin Ultrasound, 31 (2003), pp. 278-282.

[5]. Huang YK, Hsieh HC, Tsai FC, Chang SH, Lu MS, Ko PJ. Visceral artery aneurysm: risk factor analysis and therapeutic opinion. Eur J Vasc Endovasc Surg 2007;33:293-301.

[6]. H. Bergert, I. Hinterseher, S. Kersting, J. Leonhardt, A. Bloomenthal, H.D. Saeger. Management and outcome of hemorrhage due to arterial pseudoaneurysms in pancreatitis. Surgery, 137 (2005), pp. 323-328.

[7]. K. Itoh, Y. Kamiya, N. Ohno, S. Togawa, T. Nomura, H. Ohara et al. A case of pancreaticoduo-denal artery aneurysm causing pancreatic pseudotumour and duodenal obstruction. Eur J Gastroenterol Hepatol, 14 (4) (2002 Apr), pp. 457-461

[8]. N. Battula et al. True giant aneurysm of gastroduodenal artery. International Journal of Surgery, 6 (6); (2008): $459-61$.

[9]. Shanley CJ, Shah NL, Messina LM. Common splanchnic artery aneurysms : splenic, hepatic, and celiac. Ann Vasc Surg 1996;10:315-22.

[10]. Ikeda O, Tamura Y, Nakasone Y, Iryou Y, Yasuyuki Y. Nonoperative management of unruptured visceral artery aneurysms: treatment by transcatheter coil embolization. J Vasc Surg 2008;47:1212-9.

[11]. Sachdev U, Baril DT, Ellozy SH, Lookstein RA, Silverberg D, Jacobs TS, et al. Management of aneurysms involving branches of the celiac and superior mesenteric arteries: a comparison of surgical and endovascular therapy. J Vasc Surg 2006;44:718-24.

[12]. M. Ardelt, Y. Dittmar, H. Scheuerlein, U. Settmacher. A True Giant Aneurysm with a Diameter of $16.5 \mathrm{~cm}$ of the Gastroduodenal Artery with Arterio-portal and Arterio-caval Fistula - A Case Report and Literature Review. European Journal of Vascular and Endovascular Surgery, Available online 3 January 2013. 